\title{
WestVirginiaUniversity
}

THE RESEARCH REPOSITORY @ WVU

Graduate Theses, Dissertations, and Problem Reports

2021

\section{Identification of Moving Bottlenecks in Production Systems}

Funmilayo Mofoluwasola Adeyinka

West Virginia University, fma0005@mix.wvu.edu

Follow this and additional works at: https://researchrepository.wvu.edu/etd

Part of the Industrial Engineering Commons, and the Systems Engineering Commons

\section{Recommended Citation}

Adeyinka, Funmilayo Mofoluwasola, "Identification of Moving Bottlenecks in Production Systems" (2021). Graduate Theses, Dissertations, and Problem Reports. 10288.

https://researchrepository.wvu.edu/etd/10288

This Problem/Project Report is protected by copyright and/or related rights. It has been brought to you by the The Research Repository @WVU with permission from the rights-holder(s). You are free to use this Problem/Project Report in any way that is permitted by the copyright and related rights legislation that applies to your use. For other uses you must obtain permission from the rights-holder(s) directly, unless additional rights are indicated by a Creative Commons license in the record and/ or on the work itself. This Problem/Project Report has been accepted for inclusion in WVU Graduate Theses, Dissertations, and Problem Reports collection by an authorized administrator of The Research Repository @ WVU. For more information, please contact researchrepository@mail.wvu.edu. 
Graduate Theses, Dissertations, and Problem Reports

2021

Identification of Moving Bottlenecks in Production Systems

Funmilayo Mofoluwasola Adeyinka

Follow this and additional works at: https://researchrepository.wvu.edu/etd

Part of the Industrial Engineering Commons, and the Systems Engineering Commons 


\title{
Identification of Moving Bottlenecks in Production Systems
}

\author{
Adeyinka, Funmilayo
}

Problem Report submitted

to the Benjamin Statler College of Engineering at West Virginia University

in partial fulfillment of the requirements for the degree of

Master of Science in

Industrial Engineering

Bhaskaran Gopalakrishnan, Ph.D., P.E., Chair

Donald Adjeroh Ph.D., Co-chair

Thorston Wuest Ph.D.

Imtiaz Ahmed, Ph.D.

Department of Industrial and Management Systems Engineering

Morgantown, West Virginia 2021

Keywords: Shifting Bottleneck, Machine Learning, Agglomerative Clustering, Production System, Simulation 


\section{ABSTRACT \\ Identification of Moving Bottlenecks in Production Systems}

\section{Funmilayo Adeyinka}

Manufacturing sector have been plagued by bottlenecks from time immemorial, leading to loss of productivity and profitability, various research effort has been expended towards identifying and mitigating the effects of bottlenecks on production lines. However, traditional approaches often fail in identifying moving bottlenecks. The current data boom and giant strides made in the machine learning field proffers an alternative means of using the large volume of data generated by machines in identifying bottlenecks. In this study, a hierarchical agglomerative clustering algorithm is used in identifying potential groups of bottlenecks within a serial production line.

A serial production line with five workstations and zero buffer was simulated in ARENA ${ }^{\circledR}$ with data regarding blocked, producing and starvation time extracted. The extracted data was preprocessed using Python 3.7 to obtain a matrix of ones and zeros. The resultant matrix was fed into a complete linkage hierarchical agglomerative clustering algorithm to obtain clusters containing potential bottleneck workstations. Results obtained was validated using results obtained from simulation and an Elbow plot. 


\section{Dedication}

This report is dedicated to my mother, whose life has been an inspiration to me. To my sister Titilayo and her husband Mr Ajayi, on whose shoulders I stood. To my siblings Babade and Adesola, whose faith in me anchored me in tough times. To my Cousin Temitope Dere for always been there, To Babajide Ojo whose love I can always rely on and finally to my late Father, whose light shines on till now. 


\section{Acknowledgement}

I would like to express my sincerest gratitude to my research advisor and committee chair, Dr. Bhaskaran Gopalakrishnan, for his help and guidance. I also wish to acknowledge my co-chair Dr. Donald Adjeroh, for his immensely helpful suggestions and ideas. I would also like to recognize my committee members, Dr. Thorston Wuest and Dr. Imtiaz Ahmed for their assistance, support and recommendation. I would like to acknowledge Dr Christopher Roser and Dr. Mukund Subramaniyan, whose previous work was the building block for my report.

I want to acknowledge my family members, Taiwo Adeyinka, Adeyinka Babade, Adeyinka Olivia nee Adeyemo, Kayode Ajayi ,Titilayo Ajayi nee Adeyinka, Adeyinka Adesola for being there. I also want to acknowledge my best friend and best boo a girl can ever ask for, Babajide Ojo for being there every step of the way. I love you guys. I want to acknowledge my cousin Temitope Dere who is one of the reasons I am here today. I want to acknowledge my nephew Mogbekeloluwa Ajayi and my niece Adedola Adeyinka for being one of inspiration. I want to acknowledge Nyasha

Carter for being an awesome friend to me, Samuel Ogunfuye for being a great friend and to Ajibade, Taiwo for being a sister to me and lastly I want to appreciate the Redeemed Christian Church of God, Morgantown family for all the help they gave. God bless you all!!!!. 


\section{Table of Contents}

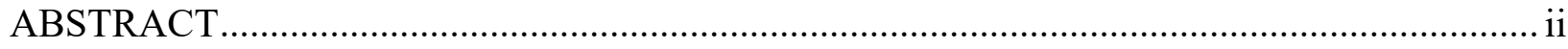

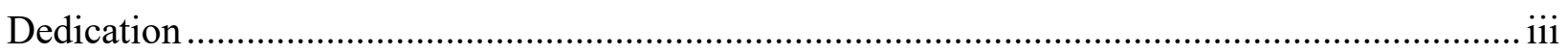

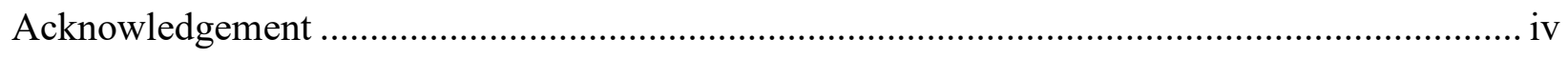

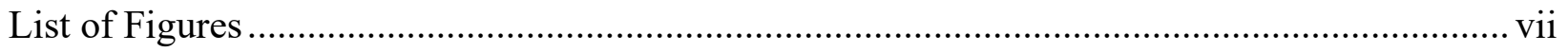

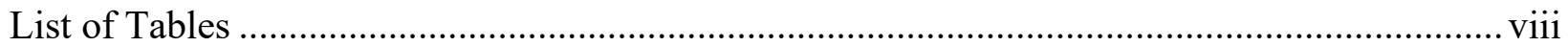

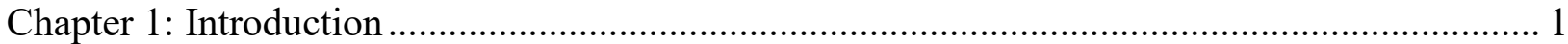

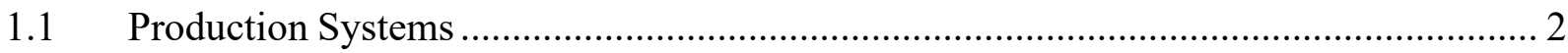

1.1.1 Continuous Production System ................................................................... 3

1.1.2 Intermittent Production System..................................................................... 3

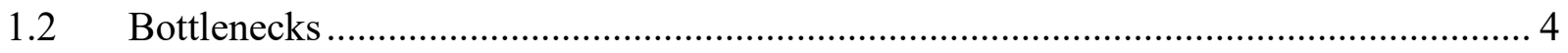

1.2.1 Terminologies Related to Bottleneck ................................................................ 6

1.2.2 Traditional Approaches to Bottleneck Control .................................................. 7

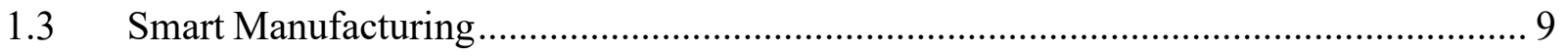

1.4 Research Motivation and Objectives.................................................................... 10

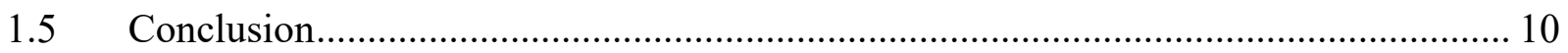

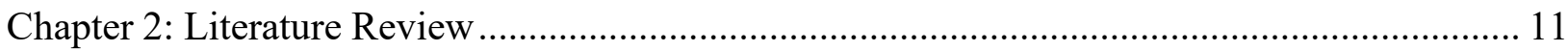

2.1 Impact of Bottleneck on Productivity and Energy Efficiency.................................. 12

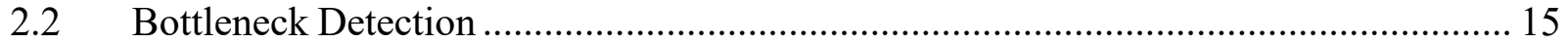

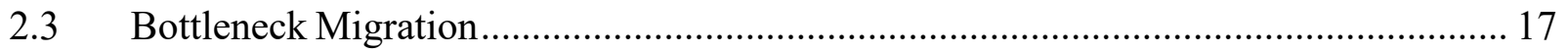

$2.4 \quad$ Bottleneck Shiftiness Control............................................................................. 19

2.5 Machine Learning Application in Manufacturing ..................................................... 20

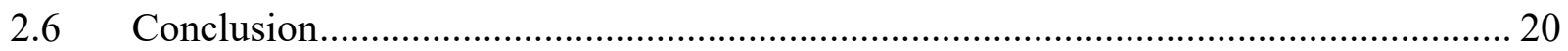

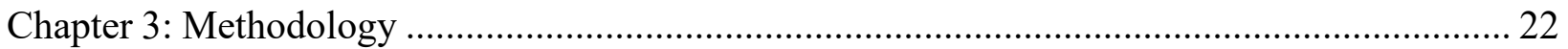

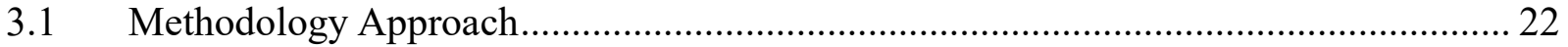

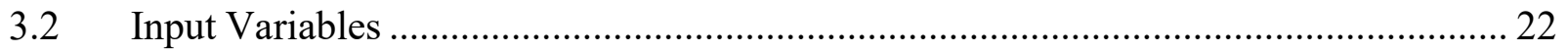

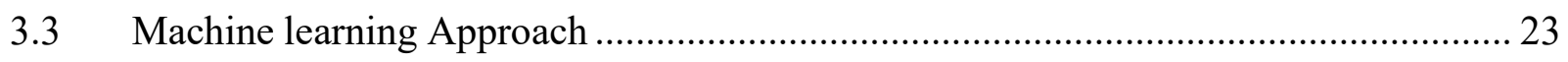

3.3.1 Data Extraction, Cleaning, and Pre-processing .................................................. 24

3.3.2 Cluster Generation and Linkage of Data ........................................................... 25

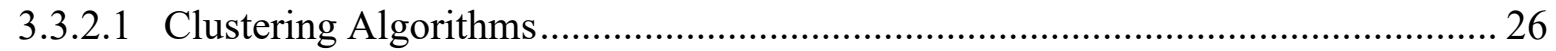

3.3.2.2 Distance Metrics for Clustering..................................................................... 27

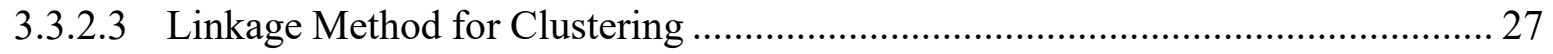


3.3.2.4 Clusters Size Determination and Cluster Variable Selection ............................ 28

3.3.3 Bottleneck Detection Machine Learning Approach........................................... 28

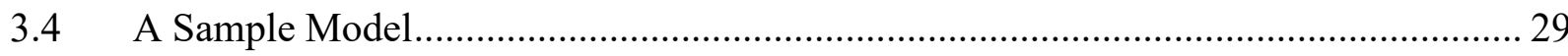

3.4.1 Data Collection, Cleaning, and Preprocessing ................................................... 30

3.4.2 Application of Agglomerative Clustering to Bottleneck Detection.......................... 30

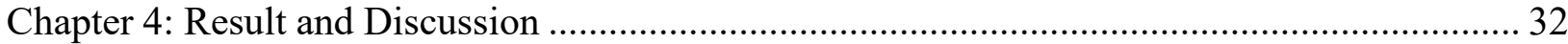

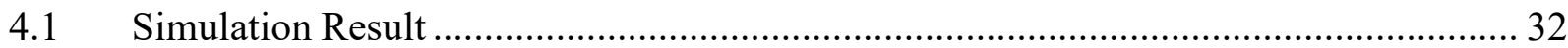

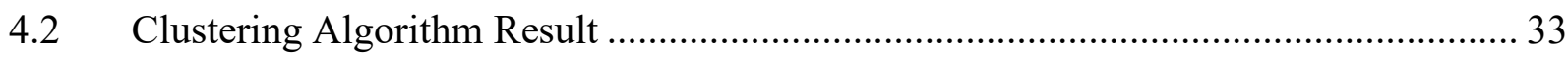

Conclusion, Future Works, and Limitation ......................................................... 36

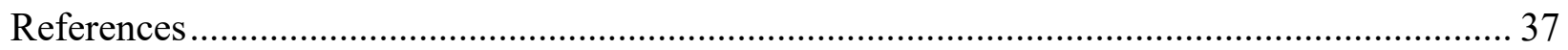

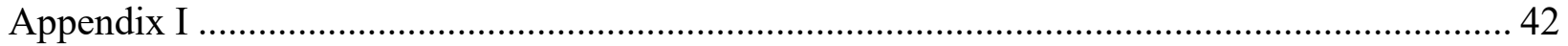




\section{List of Figures}

Figure 1.1: Schematic of a production process ............................................................... 3

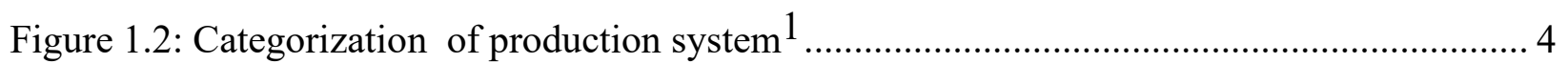

Figure 1.3: Representation of a Serial Operation with a Bottleneck......................................... 5

Figure 1.4:Schematic of Theory of Constraint Methodology [5] ......................................... 8

Figure 2.1: Graphical representation of shifting bottleneck [33] …..................................... 17

Figure 3.1: Machine Learning Workflow ........................................................................ 24

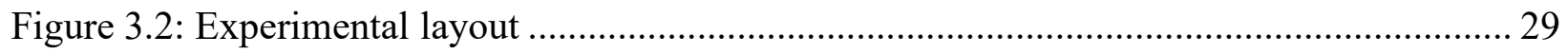

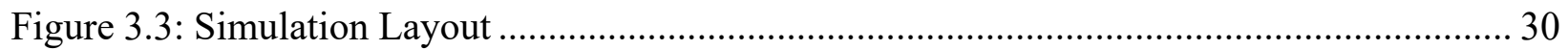

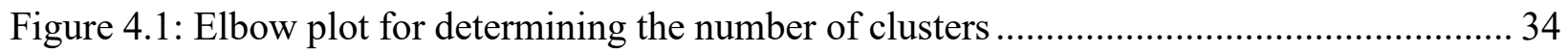

Figure 4.2: Dendrogram of machines cluster..................................................................... 35 


\section{List of Tables}

Table 3.1: Criteria for classification of event ..................................................................... 25

Table 3.2: Machine Capacity and Processing information ................................................... 29

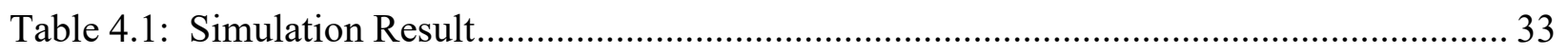

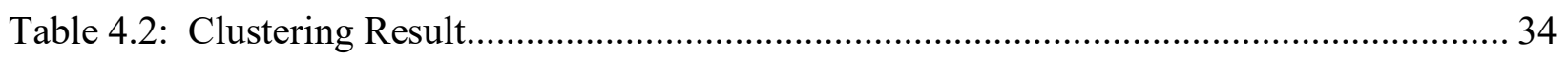




\section{Chapter 1: Introduction}

The technological advancement of the $21^{\text {st }}$ century has changed the world's economic landscape by driving globalization and industrialization while also shaping consumer behavior. The $21^{\text {st }}-$ century consumer behavior involves the demand for affordable personalized product offerings with minimal lead time; this unique requirement has led to increased competitiveness within the industrial sector with many manufacturing companies jostling to increase their market penetration while retaining their customer base. To achieve this, there is a need for companies to achieve a distinctive edge called 'Competitive Advantage' over other businesses with similar product offerings.

According to [1], Competitive Advantage can be achieved in three ways, namely:

- Competing on Differentiation: this means gaining an edge over competitors by offering unique products.

- Competing on Cost: this means gaining an edge over competitors by offering similar products at a reduced price.

- Competing on Response: is gaining an edge on competitors by offering the same products with a rapid delivery process.

However, two of the three ways of achieving competitive advantage(competing on cost and competing on response) require a tightly controlled production system with an emphasis on optimal productivity. The relationship between competitive advantage and productivity is confirmed by a study of private businesses conducted by [2], where they discovered that increase in productivity led to market growth and increased competitive advantage. Hence, improving productivity is one of the means of responding to consumer unique needs. 
Productivity in manufacturing is often measured by the throughput (although high throughput does not indicate productivity). Productivity can be increased by the minimization of input such as raw materials, labor, and/or the maximization of throughput through the judicious utilization of existing resources while maintaining a production pace that meets demand. However, machines possess finite capacity, limiting the quantity of output they can churn out, and since most production systems are like chains consisting of serial or parallel machines where processes flow from one machine to the next; the output/productivity of a production system is determined by the slowest machine as such a production system is as strong as its slowest machine. This machine(s) often is commonly referred to as the bottleneck and increased output at the bottleneck machine will often result in improved productivity for the entire production system. The impact of the bottleneck machine on productivity and by extension in meeting customer demand makes it a critical part of the production system. This means that adequate resources and attention should be allocated to it. However, allocating adequate resources to it requires that it must be correctly identified before it can be effectively managed, hence the accurate identification and prediction of the bottleneck resource(s) is the very first step in increasing productivity.

\subsection{Production Systems}

Production is the process of combining various material inputs and immaterial inputs to make an output fit for consumption (as shown in figure 1.1); in simple terms, production involves value addition to a set of inputs to achieve a predetermined output, where the outputs can be tangible (goods) or intangible products(services). 

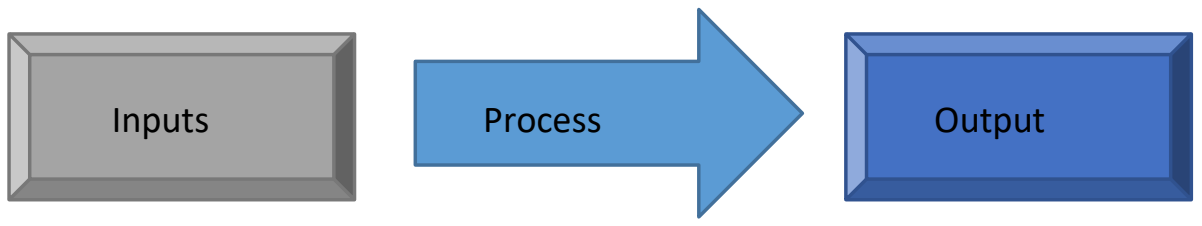

Figure 1.1: Schematic of a production process

Production system refers to the approach utilized by a business entity in its creation of goods and services and the approach is usually determined by the type of goods to be produced and the defining strategy employed by the business. Production systems when viewed as functions of process flow and demand are typically grouped into two broad categories. The production system influences both the sensitivity of the system to bottleneck and the bottleneck control strategy to be applied, for instance, a system where each process is a function of the preceding process will be more impacted by a bottleneck machine than a system where processes run independently.

\subsubsection{Continuous Production System}

In continuous systems, goods are manufactured according to forecast and the production process is carried on in a defined sequence of operations hence the presence of constraints in the loop weakens the entire process. The continuous system is further divided into two namely :

- Mass production systems

- Process production systems

\subsubsection{Intermittent Production System}

In intermittent production systems, goods are produced to fulfill the demands made by customers, and the production process is not as sequential as the continuous production system. This system allows for storage of products between processes which can act as a buffer to the bottleneck in the process. It is also subdivided into three categories, with varying levels of sensitivity to bottlenecks. 
- Job Production

- Batch production

- Project Production

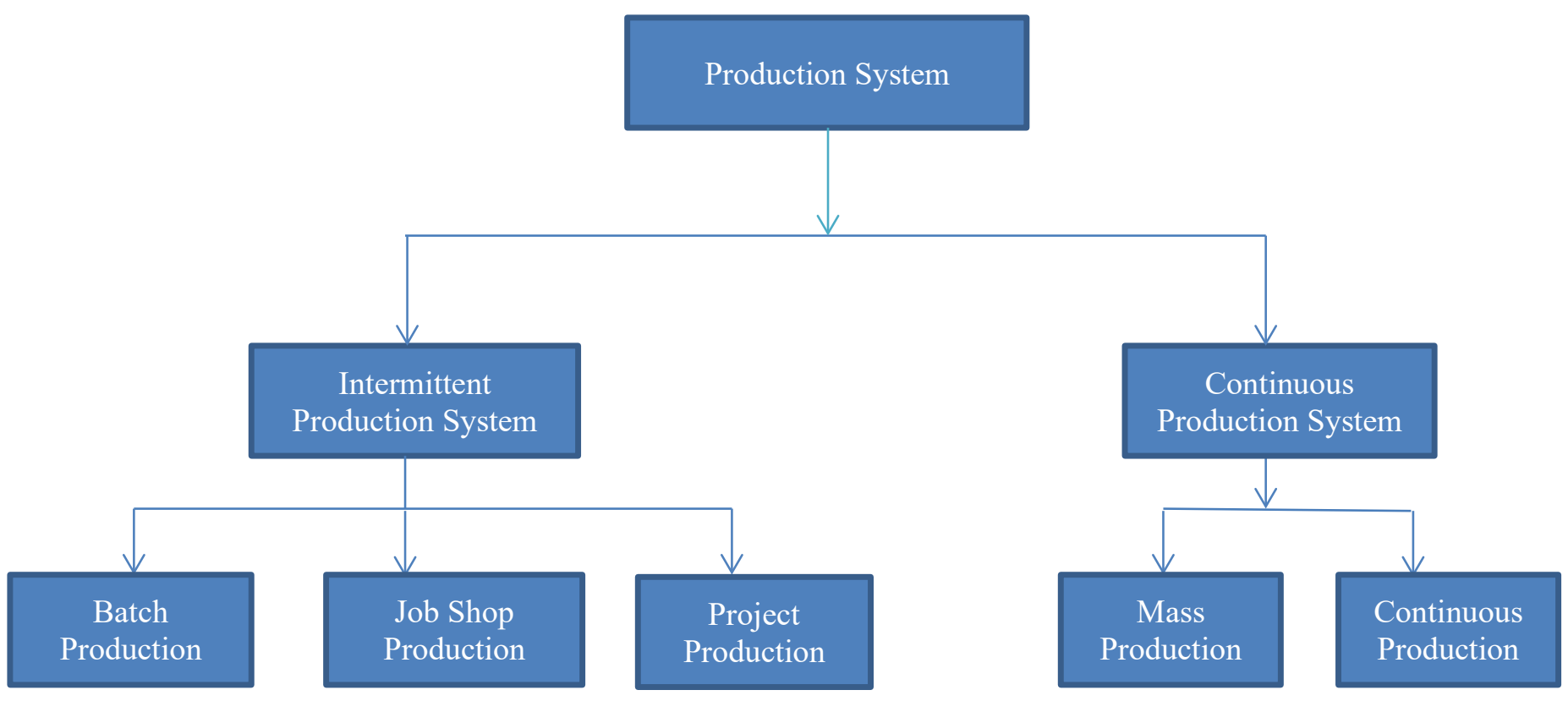

Figure 1.2: Categorization of production system ${ }^{1}$

\section{$1.2 \quad$ Bottlenecks}

A Bottleneck in its simplest definition refers to the neck of a bottle which is often the slimmest part of the bottle; that is the part of the bottle that reduces the flow of matter. ${ }^{1}$ rials down the bottle. The innate nature of resources possessing capacity and capability makes bottlenecks an unavoidable part of every production process. In a typical production process, bottlenecks are usually found in the inputs to the process. The inputs of production are conventionally classified with the acronym 5M namely Machine, Method, Man, Material, and Money, and while bottlenecks can occur along any of the $5 \mathrm{M}$, it is commonly associated with machines because machines are

\footnotetext{
${ }^{1}$ https://www.roarwap.com/pom/types-of-production-system/
} 
the actual processor of the entire system. While there are several causes of bottleneck, the simplest cause is the lack of capacity; when demand or input is greater than the processing capacity of the equipment, it becomes a bottleneck that starves and blocks the downstream equipment and upstream equipment, respectively. They can also be caused by random variations in operating conditions such as unplanned breakdowns of the machine. The impact of bottleneck on productivity is shown below. Consider the sandwich-making operation below consisting of five serial processes with constant cycle time and no buffer. Under normal operating conditions, the first completed order will be produced in 120 seconds with subsequent orders released every 35 seconds. The pace of orders released is the same as the cycle time of the toasting process making the toast the limiting process of the operation.

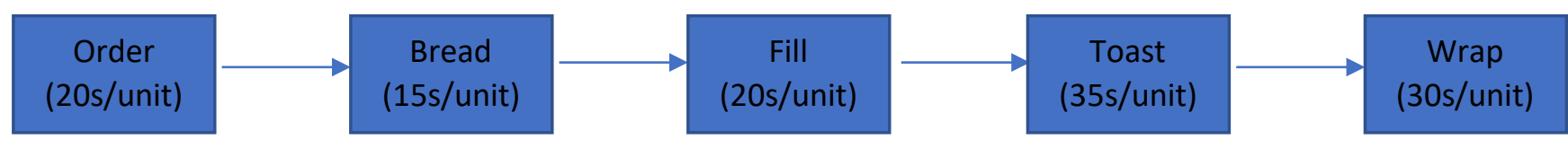

Figure 1.3: Representation of a Serial Operation with a Bottleneck

Beyond reduced productivity, a bottleneck in the system negatively affects the production plan causing the following:

- Delay in meeting customer's demand

- Wastage, and loss of quality due to delay in processing

- Increase in cost of production

The disruption caused by a bottleneck makes it the subject of concern for production managers and as result, bottlenecks resources are often the prime target of Kaizen (continuous improvement) because improvements actions on the bottleneck result in improvements on the entire production. 
However, focused control and improvement actions are often hard to implement because of the tendency of the bottleneck to shift from one machine to another during the production run.

\subsubsection{Terminologies Related to Bottleneck}

Process time: is the time it takes for one or more inputs to be transformed into a finished product or service by a process and can be obtained from the sum of times spent at different processes.

Cycle time - the cycle time can be used for a single process or the entire production process. For a single process, it refers to the time taken to process a part or unit by a machine. In terms of the entire process, it refers to the time between two consecutively produced units. The cycle time can be a constant (steady-state conditions), a variable, or completely random and it is usually determined by the potential bottleneck resource under normal operating conditions.

Buffer length: Buffers are storages that separate each machine/process and help compensate for random fluctuation in production. Buffering is often seen as a control mechanism for the bottleneck. The machine with the longest buffer length preceding it and the shortest buffer succeeding it is often seen as the primary bottleneck.

Utilization rate: This is a function of capacity and refers to the percentage of resource capacity in use. Under normal operating conditions, the resource with the highest utilization rate is the potential bottleneck of the system, however, this assumption is not always accurate.

Work in Process (WIP): it is simply defined as partially completed inventory, [3] provided a narrower and more specific definition, where WIP was defined as the inventory after the first resource and before the last resource in a production system. Bottlenecks are often associated with high WIP upstream and low WIP downstream.

Mean Time Between Failure (MTBF): the MTBF of a resource is mathematically defined as the total time of correct operation in a period divided by the number of failures; in essence, it measures 
the propensity of a resource to fail, and it is an index of the availability and the reliability of a resource. According to [4], reliability is the ability of a system or component to perform its required functions under stated conditions for a specified period while availability is the extent to which a system or component is operational and accessible when required for use. A resource that is prone to regular failure has a higher tendency to be the bottleneck of a production line under stochastic operating conditions.

Mean Time to Repair (MTTR): is mathematically defined as the total hours of downtime caused by system failures divided by the total number of failures. Like the MTBF, MTTR is also an index of reliability and availability, and a large value of MTTR is often associated with the bottleneck resource.

\subsubsection{Traditional Approaches to Bottleneck Control}

Due to the finite capacity of resources and the randomness of operations, bottlenecks cannot be eliminated in production processes, however, they can be controlled, with their effect kept within reasonable limits. Over the years, several methodologies and philosophies have arisen as a control mechanism to bottleneck, notable amongst them is the Theory of Constraints (TOC) developed by [5]. The TOC also sees bottleneck control as a continuous improvement exercise where effort is continuously expended in improving the constraint. The TOC operates on five principles which are listed below.

STEP 1: Identification of the constraints.

STEP 2: Development of a plan for overcoming the identified constraints.

STEP 3: Focus resources on accomplishing Step 2. 
STEP 4: Reduction of the effects of the constraints by offloading work or by expanding capability.

Make sure that the constraints are recognized by all those who can have an impact on them.

STEP 5: Repetition of Cycle

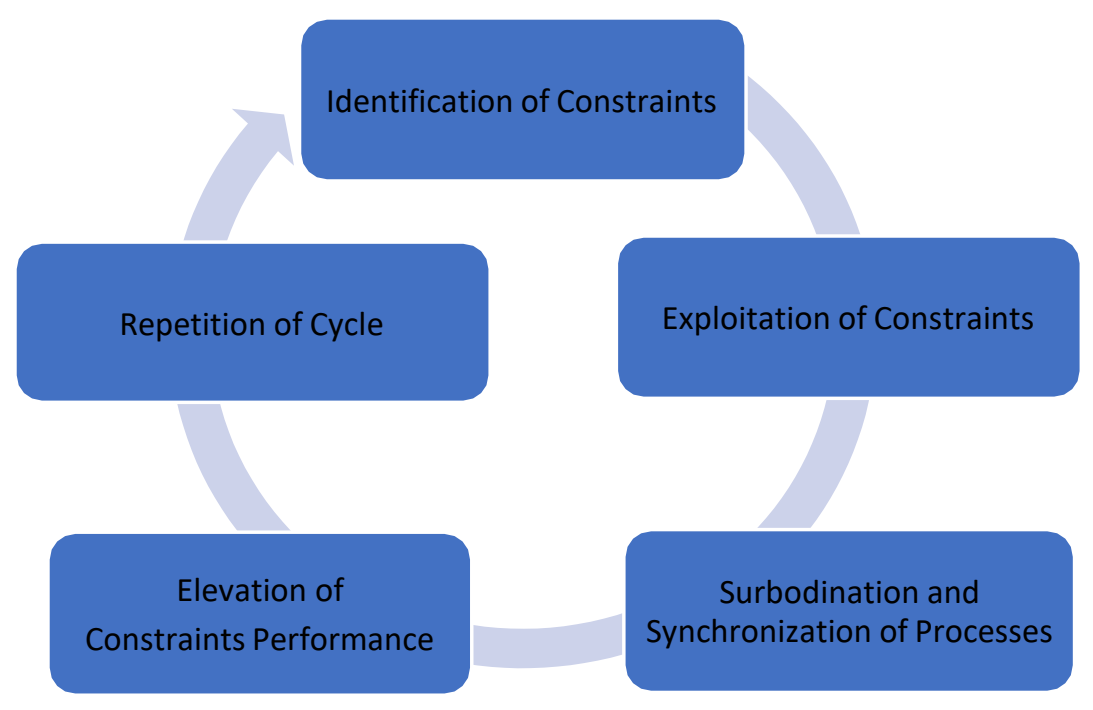

Figure 1.4:Schematic of Theory of Constraint Methodology [5]

Beyond TOC, other widely used operations management tips for bottleneck control includes:

- Reduction of downtime at bottleneck station: the sensitivity of the system to the bottleneck station means that loss time at the bottleneck has a larger effect on the entire system, hence the bottleneck station should always be active.

- Improved quality control at bottleneck station: for a production system that allows rework of faulty products. Quality is tightly controlled on the bottleneck station to avoid reprocessing. 


\subsection{Smart Manufacturing}

The global industrial sector has undergone several significant changes called industrial revolutions and these revolutions are often preceded by technological advancements. the current advancement in information and communication technology has given rise to the fourth known industrial revolution called Industry 4.0 and also called smart manufacturing (SM). Mittal et.al (2019)[6] defined smart manufacturing as a set of manufacturing practices that use networked data and information and communication technologies (ICTs) for governing manufacturing operations.

Smart manufacturing is growing in leaps and bounds for several reasons. The COVID -19 pandemic has led to faster adaptation and implementation of a highly digitalized development strategy. The Industrial Internet of Things (IIoT) and data analytics, one of the prominent bedrocks on which Industry 4.0 is premised has been leveraged in recent times in achieving smoother manufacturing operations. For instance, IIoT has led to a more connected, automated, and optimized production system and has also resulted in a more efficient supply chain by driving seamless information exchange amongst all members of the supply chain. Donnovan et.al (2015)[7] also claims that the extensive amount of data generated during production has led to an increased usage of data analytics and machine learning in the manufacturing domain to make predictions and informed business decisions.

The domain of data analytics holds great promise for manufacturing companies, with the availability of real-time data, more accurate predictions can be made, [8] has also researched the use of machine learning in the prediction of machine failure and planning of maintenanceactivities. This can also be applied in the detection and identification of bottlenecks; current bottleneck detection methods are mostly simulation-based approaches that are time-consuming, 
computationally expensive, with several limiting assumptions that fail to capture the full picture of the production system.

\subsection{Research Motivation and Objectives}

The study of bottlenecks has received an appreciable amount of research attention in previous years. However, considering the complexities of bottlenecks, most research on this topic is skewed towards long-term steady-state bottlenecking, neglecting the more realistic dynamic or shifting bottleneck. This means the impact of shifting bottlenecks on production indices is often neglected. According to [9], multi-factor productivity of the manufacturing sector decreased by $1.6 \%$ in 2019 , coupled with increased demand for shorter lead times by consumers shows the need for a more efficient and reliable production process.

The belies the need for prompt detection and prediction of the shifting bottleneck resources; adequately arming the production manager with the knowledge to allocate resources to help eliminate or elevate the current constraints. The objectives of this thesis is to:

- Apply Machine Learning algorithm in shifting bottleneck detection by generating clusters of potential bottleneck machines within a production system

\subsection{Conclusion}

The aim of every business is profitability, for businesses in the manufacturing sector profitability is causally related to productivity hence there is a growing need for efforts geared towards improved productivity. This report aims to use machine learning as a tool that helps in identifying shifting bottlenecks and in extension help production managers plan their operations and in the allocation of resources to manage the limiting effect of a bottleneck on productivity. 


\section{Chapter 2: Literature Review}

In manufacturing where production is often a result of sequential processes (machines), mostly there exists a process (machine) that determines the pace of production at each instance and such machine is often referred to as the bottleneck. Studies into the concept of the bottleneck date back to 1951, when [10] defined a bottleneck resource as a business resource that can only be used up to a certain threshold. Bottlenecks in production generated further significant research interest with the introduction of the "Theory of Constraints" (TOC) principle by [5]. However, despite the length of research, there is no universally accepted definition of a bottleneck in existent literature, mainly because bottleneck description often varies across systems, process flow, and indicators. According to [11], bottleneck definitions for a Bernoulli-modelled production system are often not

applicable to a Markovian-modelled system. Scholz-Reiter et.al (2011) [12] categorized bottlenecks according to the following indicators: relative lateness, utilization loss, work in progress, and throughput.

Lawrence and Buss (1994) [13] defined and classified bottlenecks as follows: (1) any work center temporarily under capacity, (2) the work center currently having the most jobs or customers, or (3) the work center with the greatest long-run utilization. These three definitions are also known as the "short-term", "inventory" and "production" definitions of bottleneck, respectively. Literature is replete with various definitions of bottleneck and listed below are some of the most cited definitions by other researchers.

1) The bottleneck is "the machine with the lowest isolated production rate in a system or the upstream buffer with high work-in-process (WIP) inventory" ([11]). 
2) The bottleneck is "the resource with a high ratio of sensitivity", where "sensitivity ratio" is defined as the ratio of sensitivity of a production system's performance to its isolated production rate ([11]).

3) Lowest blockage and starvation time of all the machines in a system ([14], [15]).

4) The Bottleneck is the machine to which the overall system throughput has the largest sensitivity ([16]).

These definitions are limited to static and long-term steady-state bottlenecks; however, bottleneck resources are also subject to fluctuations, shifting from one resource to another as occasioned by random variations in working conditions. In recent times, the concept of shifting bottlenecks has garnered some research interest. However, like the generic bottleneck, there is no singular definition for a shifting bottleneck. From the theory of constraints, a shifting bottleneck is often seen as the new bottleneck arising from the elevation or elimination of the previous bottleneck resource. According to [17], a single resource is a bottleneck at each instance, but then with superfluous production capacity, every resource takes turns to be a bottleneck. Lastly according to [18] shifting bottlenecks are the processes that influence the throughput of the entire system.

\subsection{Impact of Bottleneck on Productivity and Energy Efficiency}

Productivity is mathematically defined as the ratio of the output of a process to input, where input is considered as resources such as labor, energy, and raw materials. The productivity of a production line is directly proportional to its throughput, as the throughput determines the output of the line per time. The throughput of a production line is dependent on the throughput of individual resources as corroborated by [19] and specifically by the bottleneck resource as theorized by [5]. The concept of TOC is built on the relationship between bottleneck and 
productivity. Hence, bottleneck, in its simplest definition as the resource that determines the throughput, greatly influences the productivity of a production line.

According to [20], the least productive resource (bottleneck resource) determines the productivity of the entire process. They discovered that the much lower production capacity of the bottleneck resource leads to a lower utilization rate of downstream resources performing subsequent operations. Konopka (1995) [21], while investigating capacity improvement at a bottleneck resource for a semiconductor fabrication plant, discovered that reduction of efficiency loss in bottleneck resources of a semiconductor fabrication plant led to an increase in the system throughput.

Productivity improvement is achieved by either reducing input or increasing the output of the system. Efforts to increase productivity via an increment in output are mostly concentrated on the bottleneck resources; according to [22], bottlenecks are the source of interference at improving throughput and productivity. They implemented a modified form of the Theory of Constraints to elevate bottleneck resources in a heavy-duty truck lighting system plant and recorded an increase in throughput and productivity. Similar improvements were recorded in other manufacturing plants whose approach to improving productivity involved reducing the effects of the bottleneck. General Motors improved productivity with the use of software (C-MORE and TIP) that identified bottlenecks and focused efforts on bottleneck performance improvement initiatives, as bottlenecks have the greatest impact on overall system throughput ([23]). Nakata et.al (1999) [24] recorded a $10 \%$ and $2 \%$ increment in throughput and utilization and a simultaneous $13 \%$ decrease in cycle time in a micro-computer production facility, with the return of the bottleneck resource to a high performing state. 
A review of industrial applications of the TOC by [25] showed considerable improvement in productivity, inventory level, and lead time - an indication of the impacts of the bottleneck on productivity and productivity factors. [26] combined usage of constraints management and Lean manufacturing in an auto-assembly plant yielded an increase in productivity, efficiency, and the quality of output.

[27] \& [28] conducted simulations to detect bottlenecks and improve productivity. The former study recorded an $18.8 \%$ increase in productivity upon elevating the bottleneck in the simulated model of a tire-making factory, and the latter conducted a discrete event simulation for bottlenecks detection and productivity improvement for a sawmill. Results obtained after elevating a bottleneck resource showed a $25 \%$ increase in productivity.

Few literature explored the direct effect of the bottleneck on energy consumption and efficiency. According to the National Association of Manufacturers, research efforts on manufacturing energy savings and efficiency are "local in nature", focusing more on developing individual energyefficient machines and processes without considering the entire system. In a paper by [29], their research showed that shutting off idle non-bottleneck resources led to an $80 \%$ reduction in total energy consumed during idle (idleness caused by bottleneck), start-up, and shut down periods. From the data on energy consumption in manufacturing collected by [30], it is seen that significant amounts of energy are consumed during machine startup and machine idling. [29] attributed this energy consumption and waste to the blockage and starvation of machines by bottleneck resources. Hence, it can be logically concluded that the bottleneck resource contributes to energy waste and lowers the energy efficiency of a production system. 


\subsection{Bottleneck Detection}

The detection of a bottleneck resource is complicated by the inherent complexity of a typical manufacturing setup. Simple traditional detection techniques such as length of queues, average utilization rate, capacity, and inventory levels are limited, producing misleading results in several instances and failing to detect shifts in the current bottleneck. Current detection methods in most literature are either analytical- or simulation-based. However, there has been a recent shift towards a data-driven approach. In literature, not all proposed methods attempt to instantaneously detect the bottleneck. Some detection methods tend to be predictive, such as the machine learning model of [31]. Analytical methods do not scale well for short-term bottlenecking, being more applicable to a long-term bottleneck. The oversimplification of the system's complexity limits the analytical detection of bottlenecks, the assumptions and approximations lead to loss of accuracy. A simulation-based method, while a useful tool for shifting and momentary bottleneck detection, suffers from a huge computational requirement.

Several methods applicable to both average long-term bottleneck and shifting bottleneck have been proposed. Lawrence and Buss (1994) [32] with the assumption that all resources are potential bottlenecks and the Jackson network production model, considered the utilization rate and the probability of resources to be the bottleneck. The authors devised a bottleneck shiftiness measure $(\beta)$, which shows the tendency of a bottleneck in a system to shift. While this method does not identify the shifting bottleneck, it provides a starting point.

$$
\beta=1-c_{v} / \sqrt{n}
$$

$\mathrm{C}_{\mathrm{v}}=$ coefficient of variation of the bottleneck probability of machines

$\mathrm{n}=$ number of machines 
[24] introduced a workflow control system for a semiconductor manufacturing plant called "JUSTICE/MORAL" ("just time process control system/method of optimum-buffer restriction and adjustment logic") which dynamically detects the bottleneck resource, feeding work to theresource as needed. The active duration method proposed by [33] is used for both steady-state andnonsteady-state systems. A resource is defined as active whenever the resource causes other resources to wait, with the active period including process time, repair time, and service time. Theresource with the longest active duration at any instant is the momentary bottleneck and the overlapof the active period of a bottleneck with the previous or subsequent bottleneck represents the shifting of the bottleneck. Active duration for all resources is recorded and the average bottleneckand shifting bottleneck is determined from the record. Wang et.al (2011) [34] modified the activeduration method proposed by [33] with the inclusion of throughput to accommodate the economic implications of the bottleneck resource. Tamilselvan et.al (2010) [35] defined a bottleneckresource as the resource that renders other resources inactive and proposed the "inactive duration method" as an offshoot of the active duration method. The time-record of all active and inactive duration of each resource is obtained and analyzed. The sole and shifting bottleneck is obtained using the concept of the active duration; however, the inactive duration becomes the indicator. Subramaniyan et.al (2018) [36] considered utilizing software resources such as Manufacturing Execution System (MES) in the detection of the bottleneck. The approach involved the extraction of data from MES and the development of a bottleneck detecting algorithm based on the active period method. 


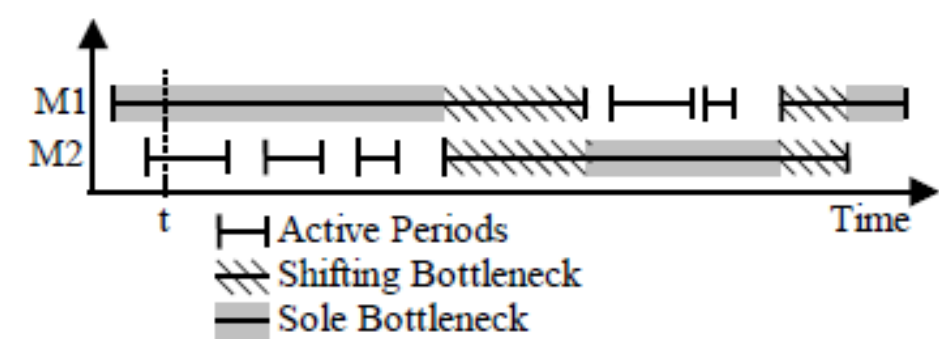

\section{Figure 2.1: Graphical representation of shifting bottleneck [33]}

[16] utilized the concept of blockage and starvation to develop the "turning point method". "Blockage" is defined as a state where a resource blocks the upstream resource, while "starvation" is defined as the state where a resource starves the downstream resource. The "turning point" is determined to be the point in the system for which the starvation duration becomes greater than the blockage duration. If no turning point is found, the first resource becomes the bottleneck if the starvation duration of the system is greater than the blocked duration, and the last resource is the bottleneck if blockage time exceeds starvation time. A bottleneck prediction method for a stochastic production system was proposed by [37], this method was based on the autoregressive moving average (ARMA) model. The blockage and starvation data are converted into a timeseries, the prediction algorithm is subsequently modeled using ARMA while [18] introduced the bottleneck walk to detect both shifting and average bottleneck in a production system. The bottleneck walk is based on observations of different process and inventory states on the shop floor and is applicable applies to both manual and automatic processes.

\subsection{Bottleneck Migration}

According to [13], a bottleneck shift is mainly caused by random variation in a production system. They implied an existing relationship between the average utilization rate of resources in a production system and "bottleneck shiftiness", theorizing that shifts in the bottleneck would be 
maximized when all work centers' utilization rates are equal, as every work center is equally probable to be the bottleneck.

Building on the research of [32] and analyzing the effects of various production parameters (such as arrival rate, service rate of bottleneck and non-bottleneck resource, type of shop (flow or batch), and bottleneck position in the shop) on bottleneck shiftiness. Using multiple regression analysis, it was concluded that increasing either the arrival rate or the processing time at the bottleneck leads to a decrease in bottleneck shiftiness, meaning the bottleneck will be more constant. However, increasing the number of work centers (size of the shop) leads to an increase in bottleneck shiftiness. According to [39], for a system without a specific bottleneck and non-bottleneck, bottleneck shiftiness is caused by the change in a system's operating parameter. Product mix, production batch lot size, and load-balanced level were presumed to be probable causes of bottleneck shiftiness by [40] \& [41], with [41] further categorizing the causes of bottleneck shiftiness into two, namely demand fluctuation and facility exception causes. They opined that changes in product mix due to changes in customers' orders lead to changes in production resource load. A new product mix with balanced resource utilization tends to increase the bottleneck shiftiness of the system. This view is in tandem with [32] speculation about the relationship between bottleneck shiftiness and utilization rate. Facility exceptions such as machine failure, material shortage, and operator error can cause a shift in the bottleneck; The failure of a nonbottleneck resource with a suboptimal buffer size can halt production and such a resource becomes the temporary bottleneck. The effect of learning and forgetting on bottleneck shift in a two-stage production system was investigated by [42], and they concluded that bottleneck shift may occur when an initially slower stage overtakes a previously faster stage over time due to a higher learning rate of operators. 


\subsection{Bottleneck Shiftiness Control}

The goal of every bottleneck detection attempt is to develop management and control measures that reduce bottlenecks and improve productivity. According to [32], bottleneck shiftiness can be reduced while simultaneously improving flow time performance by increasing the capacity of nonbottleneck work centers, resources with low long-run utilization levels. This approach tends to negatively impact the mean flow times. [40] proposed three solutions to solve bottleneck shiftiness, each a function of the underlying cause. They suggested the TOC concept of [5] to solve shiftiness as caused by product-mix, the maximization of process batching and the simultaneous minimization of transfer batching. This approach seeks to reduce the production lead time and control bottleneck shift caused by production batch lot size, as well as the adoption of buffer management as a tool to prevent bottleneck shiftiness with unknown causes.

To combat the detrimental effects of shifting bottleneck on energy consumption, [43] proposed a heuristic for real-time electricity demand response which considers the instantaneous throughput bottleneck detected using the turning point method of [16]. The heuristic determines the maximum time window for switching off energy, stating that the non-bottleneck resource with the highest blockage and starvation time should shut off whenever energy consumption exceeds a preidentified limit; Shutting off energy to the specified resource during the time window had no corresponding negative impact on the system's throughput. [44] investigated energy-saving opportunities for automotive manufacturing systems using a serial production line. While considering the stochastic nature of the production system, they developed a systematic datadriven method of bottleneck records that quantifies an "energy-saving opportunity" (ESO) without negatively impacting productivity. 


\subsection{Machine Learning Application in Manufacturing}

Machine learning (ML) is often defined differently by various classes of people; however, a generally accepted definition is that it is a subset of artificial intelligence where computers are programmed to learn automatically. ML has been applied in a wide range of sectors, ranging from entertainment, health, financial services etc. to solve various business problems and it has also been steadily used in the manufacturing domain. According to [45], the dynamic operations of the manufacturing domain and the np-hard nature of its optimization problems make it a viable candidate for machine learning functionalities. There exist various problems in the manufacturing domain where ML holds great promises; current areas where ML has been utilized in manufacturing include quality inspection, fault localization, throughput prediction, and fault prediction with various literature existing on the application of ML in solving problems in the manufacturing space. Convolutional Neural Network was used to predict wear in in-process tools by [46] while [47] built soft sensors using a deep neural network (DNN) algorithm to predict the cut-point temperature of heavy diesel in a crude distillation unit. Support Vector Machine was used by [48] to diagnose tool breakage fault in a face milling process under varying cutting conditions. However, there still exist numerous opportunities that ML can explore in the manufacturing domain

\subsection{Conclusion}

The literature review shows that while there has been significant research on bottlenecks in general. Research into moving bottlenecks has been few and in between. The research into moving bottlenecks is also either limited to detecting or predicting the shift in bottlenecks using either simulation or a machine learning algorithm. Previous research also fails to combine the predicting 
process with determining causal factors. This problem report aims to address these gaps by detecting potential moving bottlenecks using both simulation and machine learning algorithm. 


\section{Chapter 3: Methodology}

This problem report aims to identify potential shifting bottlenecks in a production system by simulation and compare with an agglomerative hierarchical clustering algorithm experimented with by [49]. The input information for the simulation will be the type of production system, number of machines, cycle time, and the inter-arrival time between two consecutive inputs.

\subsection{Methodology Approach}

A serial line containing five machines with constant arrival time, constant processing time with no downtime will be developed using ARENA $^{\circledR}$ simulation software. The process flow is described below.

Step 1: creation of a simulation of a serial production line with five machines, zero buffers and constant processing time in ARENA $^{\circledR}$.

Step 2: extraction of event log and generation of the active and inactive period profile for each machine for the predefined time interval using the state specified in fig 3.1.

Step 3: Application of agglomerative clustering algorithm to generate bottleneck clusters within the production system.

Step 4: Analysis and verification of result obtained by the agglomerative hierarchical clustering algorithm.

\subsection{Input Variables}

The input variable refers to the parameters describing the operations and state of machines in the production system. The specific parameters are explained in detail below. 
Process time: refers to the time required to process a unit by a machine. The processing time can be modeled by different statistical distributions as it may be a constant, a variable, or random. For large volume production such as continuous production systems and assembly lines, the processing time is approximately constant.

Inter-arrival time: refers to the time difference between the arrival of one job and the arrival of the next job. The inter-arrival time can be a constant or can follow a normal distribution, however, it is mostly exponentially distributed.

Downtime: refers to the duration in which a machine is not producing due to its failure. It does not include time spent waiting for parts from other machines. The downtime consists of both the duration of failure and the duration of the repair. The downtime in this thesis will be constant for some cases, while some will have an exponential distribution/normal distribution. The downtime at every instance will be obtained from the mean time to repair (Mean time to repair: defined as the average time necessary to troubleshoot, remove, repair, and replace a failed system component).

\subsection{Machine learning Approach}

The machine learning approach works with data generated from the simulation and the program will run in Python 3.7 environment. The workflows are described below. 



Figure 3.1: Machine Learning Workflow

\subsubsection{Data Extraction, Cleaning, and Pre-processing}

The input data for the machine learning algorithm will be the data extracted from the ARENA $^{\circledR}$ simulation. The quality of the output is significantly correlated to the quality of the input data; hence, the extracted data will be cleaned. Cleaning will be done using Python. The variable of interest is the total active time of each machine for a specific production run. The data preprocessing step includes the classification of the events into active duration and inactive 
duration in accordance with the rules shown below. Upon completion of the classification process, the active time duration of each machine will be summed for each production run to generate a time series $\mathrm{M}$ by $\mathrm{N}$ matrix.

Where:

$\mathrm{M}=$ number of machines

$\mathrm{N}=$ length of production runs

Table 3.1: Criteria for classification of event

\begin{tabular}{|l|l|l|}
\hline Event Name & Event Classification & Classification \\
\hline Breakdown & A machine is down due to a fault & Active \\
\hline Production & Machine is currently produced & Active \\
\hline Blocked & Machine is not producing due to issue from downstream machine & Inactive \\
\hline Starved & Machine is not producing due to issue from upstream machine & Inactive \\
\hline Repairs & A machine is not producing due to unplanned repairs & Active \\
\hline
\end{tabular}

\subsubsection{Cluster Generation and Linkage of Data}

The bottleneck in an active producing system rarely stays constant, shifting randomly or in a specific format across some machines, the active duration data of each machine while sufficient to detect the momentary bottleneck and the most consistent bottleneck is insufficient to detect the shift in the bottleneck. Hence, it is necessary to group the machines together based on their tendency to experience a bottleneck shift. Traditionally, the shift can be identified by plotting the graph of the active and inactive period of each machine together, however, for the proposed machine learning approach, the only known variable is the active period and the tendency of shift 
is unknown; this unknown relationship can be identified using a clustering algorithm. Clustering algorithms are unsupervised algorithms with no defined variable of interest, with the predictor variables clustered/ linked together according to some predefined metric such that variables within clusters are more closely related to each other than variables in different clusters.

\subsubsection{Clustering Algorithms}

Clustering algorithms can be classified into several categories namely: partitioning, hierarchical, graph-based, model-based, and density-based clustering with the hierarchical and partitioning clustering method being more popularly used. The hierarchical clustering method is majorly divided into agglomerative and divisive hierarchical clustering while K-means is the more popularly used method for partitioning clusters. Though the k-means is more computationally efficient, the agglomerative hierarchical method will be used because K-means requires the number of clusters as an input, however, this itself is the output of interest. K-means is also more suited for large datasets, the dataset will be of medium size and agglomerative clustering will scale well. Lastly, the agglomerative hierarchical clustering method has been successfully used by [49] in detecting bottlenecks and by [51]) in scheduling bottleneck clusters in job-shop type production systems. The algorithm is initialized with each machine acting as individual clusters with their distance with respect to each other calculated. The clusters are then fused to form a bigger cluster based on a linkage criterion with respect to the calculated distance measure until all clusters are merged into one cluster. The resulting cluster tree is referred to as the dendrogram and it shows the sequence and the point where a cluster merges with the next respective cluster. This approach has some disadvantages that are listed below:

- It does not offer the best result with an increase in the number of analyzed variables. 
- This algorithm is a greedy one, hence there is no way to correct the wrong clustering of variables made at the initial stages of the procedure

- It is computationally expensive

\subsubsection{Distance Metrics for Clustering}

The input dataset to the clustering algorithm is an $\mathrm{M}$ by $\mathrm{N}$ matrix showing the active duration of each machine for a particular period. There is a need to develop a metric that shows the similarities and dissimilarities between the machines in the system. The chosen measure forms the basis for clustering. Common distance measures used in time series clustering are Euclidean distance, Manhattan distance, Mahalanobis distance, and more recently the Dynamic Time Warping (DTW) distance. The Euclidean distance will be used as the distance measure because there was no stochastic element considered in the set-up. The bottleneck can shift during a single production run but the bottleneck group will remain the same across several production runs, this makes the Euclidean distance a good distance metric as it performs one-to-one mapping.

\subsubsection{Linkage Method for Clustering}

The fusion of clusters is based on the shortest distance between existing clusters as decided by a linkage criterion, for an agglomerative hierarchical clustering method, there are seven commonly used linkage criteria namely: Single linkage method, Complete linkage method, Average linkage method, Weighted pair-group average method, Centroid method, Method of weighted centroids and Ward method of minimum variance. The choice of linkage criterion is important because it has a profound impact on the quality and accuracy of the clusters developed. The complete linkage method which works by forming clusters based on the farthest distance between variables is recommended by [52] for cases where the variables form well separated and compact clusters, in actual production systems, bottleneck shift will be more consistent within some subset of machines 
compared to another subset for different production runs making the complete linkage method a suitable one. It was also discovered by [53] that the complete linkage method is more suited to the DTW distance metrics than the single complete linkage.

\subsubsection{Clusters Size Determination and Cluster Variable Selection}

The aim of this step is to determine the optimal number of clusters and the machines associated with each cluster from the dendrogram obtained from the previous step. There are several methods in determining the number of clusters such as the elbow method, silhouette method, within clusters sum of squares, and between clusters sum of squares (BCSS) method. The elbow method is the most popular method; here the user looks for the point where the slope changes from being steep to being shallow (it gives a graph that looks like the human arm-elbow-hand connection), this method combined with the dendrogram will be used as to determine the numbers of clusters. Upon the conclusion of cluster size, each variable (machine) will be allocated to its respective clusters.

\subsubsection{Bottleneck Detection Machine Learning Approach}

The shifting bottleneck and the probable primary bottleneck detection method would also be graphical. However, the data to be plotted would be estimated from the clusters determined in the previous step. For each cluster, the average active duration for the machines in such cluster for each production run will be computed and plotted. The resulting graph would be visually inspected to ensure the distinction of time series across clusters. The failure of the visual check will result in the repetition of the cluster determination and selection process. The failure of the visual check will result in the repetition of the cluster determination and selection process. The success of the check would result in further graphical analysis of the set of machines within the cluster. The machine with the highest average active period will be regarded as the primary bottleneck and the 
machines within the cluster with the highest average active period will the regarded as the set of shifting bottlenecks.

\subsection{A Sample Model}

A simple serial production line with five machines and zero buffer as shown in fig. 3.2 was modeled using the ARENA simulation software. The sample model was inspired from an example in chapter $7 \mathrm{~b}$ of [1] and from an existing ergonomic line set-up in Nigeria Bottling Company.The ergonomic line consisted of three individual bottling machines coupled together to run as a block. The cycle time across all machines was constant, the production process was modeled suchthat inputs only arrive when the first machine is free. The simulation ran for an hour with zero breakdowns and zero maintenance activity. Dual capacitated machines can process two products simultaneously and are assumed to be active when it is producing at least a product.

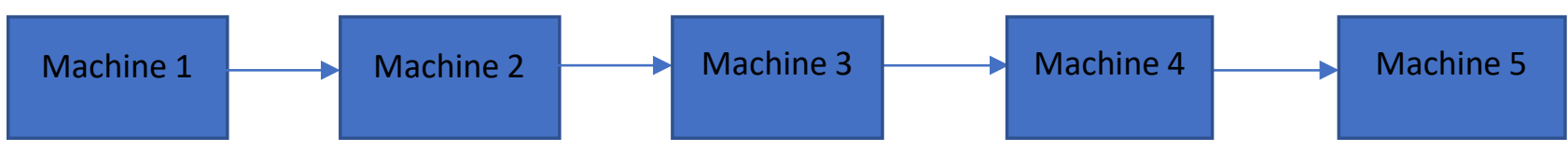

Figure 3.2: Experimental layout

Table 3.2: Machine Capacity and Processing information

\begin{tabular}{|l|l|l|}
\hline Machine & Capacity & 30 secs \\
\hline Machine 1 & 1 & 15 secs \\
\hline Machine 2 & 2 & 20 secs \\
\hline Machine 3 & 2 & 40 secs \\
\hline Machine 4 & 2 & 37.5 secs \\
\hline Machine 5 & 1 & \\
\hline
\end{tabular}




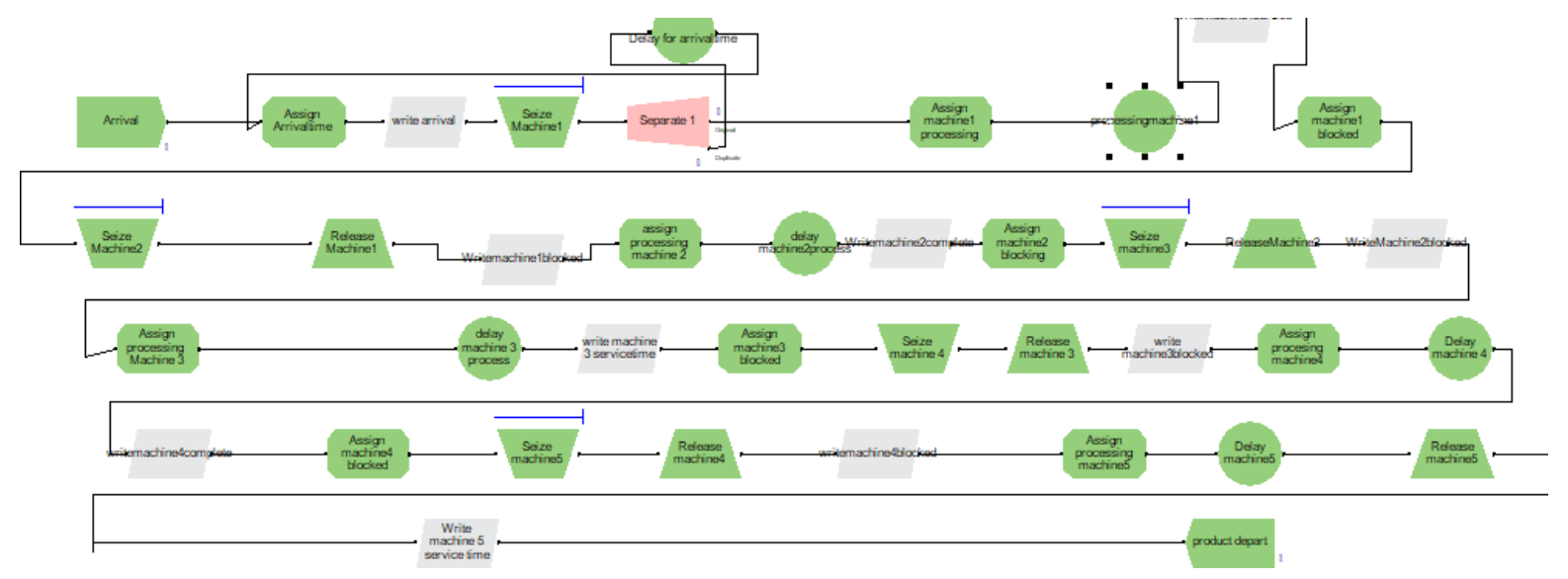

Figure 3.3: Simulation Layout

\subsubsection{Data Collection, Cleaning, and Preprocessing}

Time series of each primary event was gathered from the simulation (primary event is defined as the event that changes the state of the system, for example, service completion at a machine, blockage time). Preprocessing operations to extract the time series of the state of the machines from the raw time series file obtained from the simulation was carried out in python using the predefined metrics in figure 3.1. The result of the preprocessing stage is a comma-separated-values (CSV) file shown in Appendix 1. Appendix 1 shows that at 270 seconds, all machines in the system were processing a product, however at 275 seconds, machine 3 has become inactive, this means either machine 3 is being starved by machine 4 or it has been blocked by machine 2 .

\subsubsection{Application of Agglomerative Clustering to Bottleneck Detection}

The output of the preprocessing stage was taken as the input of this stage, the CSV file was converted to an $\mathrm{m}^{*} \mathrm{n}$ matrix where:

$$
\mathrm{m}=5 \text { (number of machines) }
$$


$\mathrm{n}=1440$ (state of the machine at each time interval)

The resultant matrix was fed into a SCIPY agglomerative clustering algorithm. The Euclidean distance was used as distance criteria because the machines operated under constant conditions and will output the same result for n number of replications. For this thesis, the complete linkage criteria will be used however for the purpose of the sample model, four linkage criteria namely: complete linkage, average linkage, single linkage, and ward linkage were used to construct four dendrograms. The dendrogram is a visual tree that aids in seeing where the individual machines merge with another and the most similar machines. An elbow plot is constructed to act as a guide in determining the number of clusters. The output of this process was the number of clusters among the machines and the machines in each cluster. Machines in the same clusters as the primary bottleneck represent the other bottleneck in the system. 


\section{Chapter 4: Result and Discussion}

This chapter contains the result of the simulation and the agglomerative hierarchical clustering algorithm discussed in chapter 3 . The machine learning algorithm ran on a 12GB RAM quad-core Intel i-5 1035G1 local machine. The execution time for the data cleaning and preprocessing step was $34.6 \mathrm{~s} \pm 7.28 \mathrm{~s}$ per loop, the execution time for the cluster generation step was $69.3 \mathrm{~ms} \pm 5.07$ $m s$ per loop and the execution time for the cluster size determination step was $171 \mathrm{~ms} \pm 10.1 \mathrm{~ms}$ per loop.

\subsection{Simulation Result}

The bottleneck of a system is defined as the machine that determines the cycle time of the production system. In the sample model, a product is released from the system every 37.5 seconds which is the processing time of the last machine in the system. At the end of 3600 seconds, 101 products had entered the system, of which 93 had undergone complete processing and exited the system. Machine 4 had the highest active period because it was always producing

However, for the first 10 minutes of the simulation, Machine 1 had the longest active period at any instance making it a momentary bottleneck, at 105 secs the bottleneck started shifting from machine 1 to machine 5 as seen in the time series of the active period contained in Appendix I. Machine 1 was the primary starving machine, starving Machine 2 while Machine 5 was the primary blocking machine blocking all other machines in the system. All other information such as the average time spent in the system is given below.

The simulation shows that while there are primary bottlenecks in a system, there exist secondary bottlenecks within the system and that the bottleneck at any instant can change from one machine to another. 
Table 4.1: Simulation Result

\begin{tabular}{|l|l|}
\hline Simulation result & \multicolumn{1}{l|}{ Time } \\
\hline Average time spent in System & 287 secs \\
\hline Value-added time & $142.5 \mathrm{secs}$ \\
\hline Percentage active time (Machine 1) & 0.835531 \\
\hline Percentage active time (Machine 2) & 0.41499 \\
\hline Percentage active duration (Machine 3) & 0.541291 \\
\hline Percentage active duration (Machine 4) & 0.981957 \\
\hline Percentage active duration (Machine 5) & 0.970854 \\
\hline
\end{tabular}

\subsection{Clustering Algorithm Result}

The defining height of the dendrogram for determination of cluster size was chosen to be fifteen, at this height, the number of clusters corresponds to 3 . This choice of cluster size was validated using an elbow plot. The y-axis of the elbow plot in figure 3.5 indicates the variance within clusters and the plot shows that the cluster size that minimizes variance is cluster size 3 and cluster size 4. The height of the dendrogram shown in figure 3.6 represents the distance between cluster nodes and shows the point of convergence for the different machines in the system. From the dendrogram, machine four is more like machine five that the other machines in the system and machine 
three is more akin to the cluster of machine four and machine five. Hence, it is likelier than the bottlenecksshift between these three machines as shown by the simulation. The sample model considers static operating conditions where productions run at different periods behave alike; however, Effectiveness of the algorithm might decrease if used to cluster machines operating under dynamic conditions because random failures might occur, and machines will behave differently across different production runs. The potential drop in effectiveness will be because of the distance metric used in the algorithm. The current algorithm uses the Euclidean distance as it distance metric, the metric performs one-to-one mapping and might fail to match machines with identical profile across different production runs. To avoid the drop in effectiveness, the Dynamic time warping(DTW) is more suited to dynamic conditions as it performs one-to-many mapping.

\section{Table 4.2: Clustering Result}

\begin{tabular}{|l|l|}
\hline Clusters & Machine (s) in Cluster \\
\hline Cluster 1 & Machine 5, Machine 4, Machine 1 \\
\hline Cluster 2 & Machine 2 \\
\hline Cluster 3 & Machine 3 \\
\hline
\end{tabular}

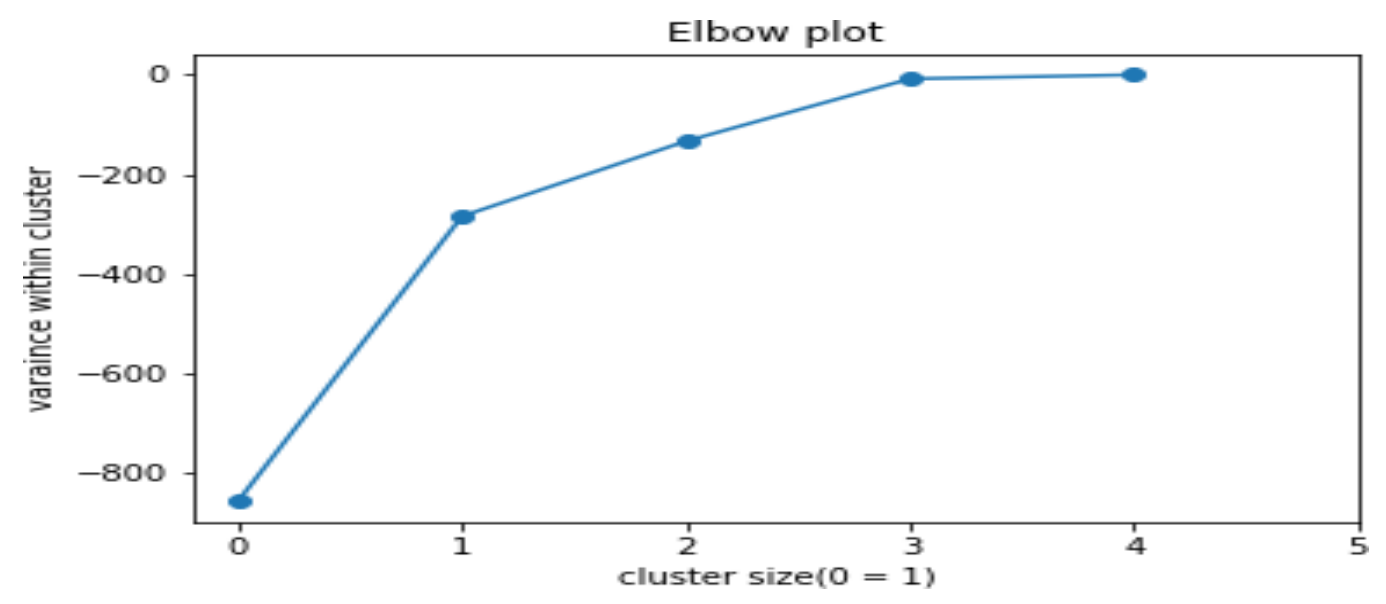

Figure 4.1: Elbow plot for determining the number of clusters 


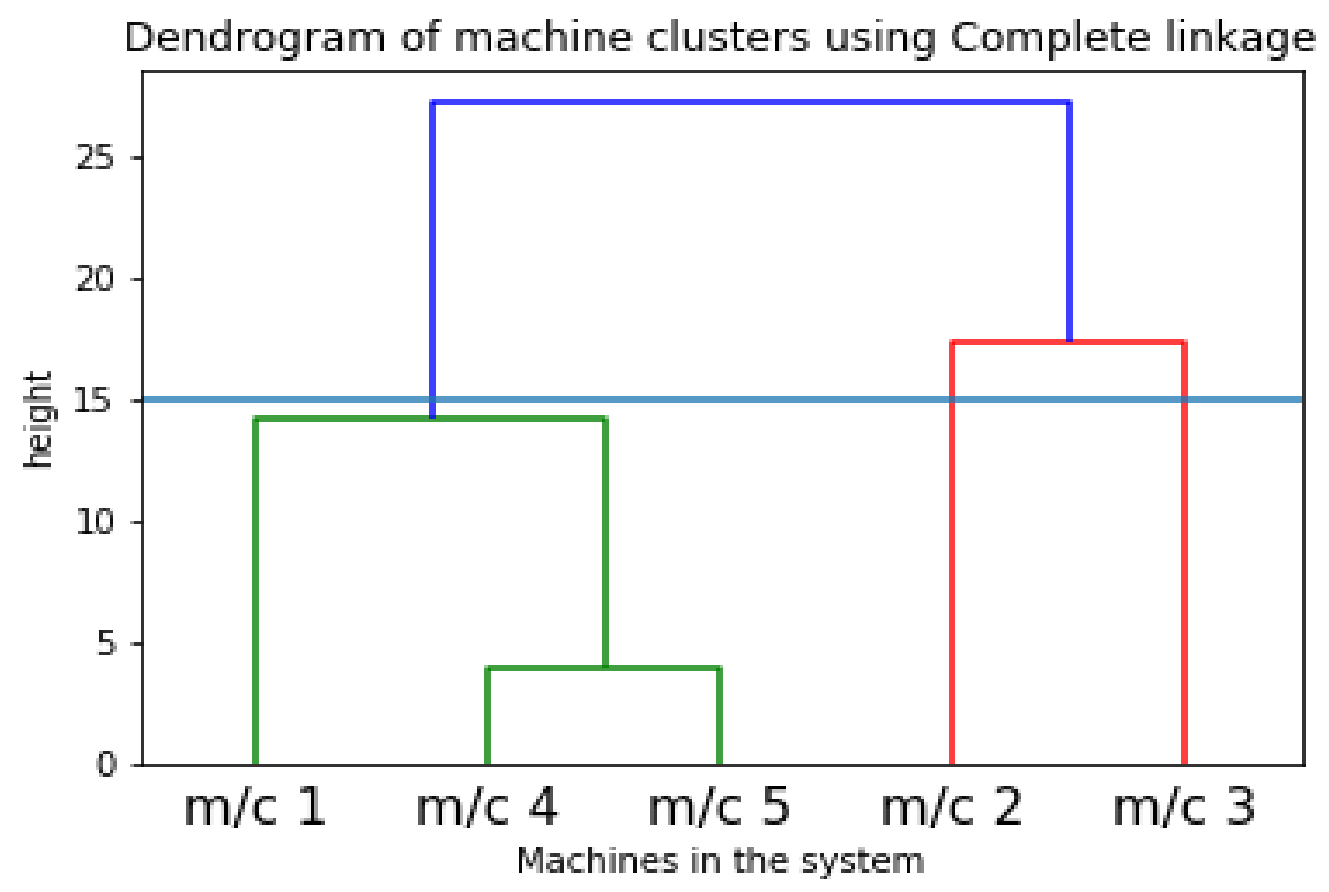

Figure 4.2: Dendrogram of machines cluster

A production system in real-time is complex and has a high degree of fluctuation, capturing all the intricacies complicates the problem, tending towards a tangled mess. To simplify the model, the assumptions listed below will be made. There also exist limitations to the proposed simulation model.

- The production system is assumed to be discrete

- The work units arrive individually and are processed individually

- Work is processed on First Come First Serve Basis (FCFS)

- The First Machine is never starved, and the last machine is never blocked

- The machines can be in different states either: producing, starved, blocked, failed, or being repaired

- The active period of the machines is uncorrelated across time periods in the production run. 


\subsection{Conclusion, Future Works, and Limitation}

From the result obtained, the use of machine learning algorithms shows great promise in the detection of shifting bottlenecks, however more improvements need to be done. The problem report was limited by the unavailability of real life, as this made it impossible to assess the performance of the algorithm in real time.

Future research might include extending the algorithm to non-serial production with stochastic operating conditions such as random breakdowns, random processing time and random interarrival time. The research can also be extended to include the development of a measure called bottleneck shiftiness probability for each machine. This measure can be utilized in predicting the next bottleneck resource within the system. Thirdly, the use of an alternative clustering algorithm such as density-based spatial clustering of applications with noise (DBSAN) can be explored and its performance compared to current algorithms used. Lastly, research can be conducted on integrating the algorithm into a production line for the generation of real-time update and feedback. 


\section{References}

[1] Heizer, J., Barry R., and Chuck M. 2017. Operations management: sustainability and supply chain management

[2] https://www.bizjournals.com/bizjournals/news/2019/06/25/productivity-can-create-a-strongfoundation-for.html

[3] Conway, R., Maxwell, W., McClain, J. and Thomas, L. (1988). The Role of Work-In-Process Inventory in Serial Production Lines. Operations Research. 36. 229-241. 10.1287/opre.36.2.229.

[4] Torell, W and Avelar, V. (2004). Mean Time Between Failure: Explanation and Standards.

[5] Goldratt, E. (1990): Theory of Constraints, New York: North River Press Inc.

[6] Mittal, S, Khan, M., Romero, D., and Wuest, T. (2019). Smart Manufacturing: Characteristics, Technologies and Enabling Factors. Proceedings of the Institution of Mechanical Engineers Part B Journal of Engineering Manufacture. 233. 1342-1361. $10.1177 / 0954405417736547$.

[7] O’Donovan, P., Leahy, K., Bruton, K., \& O’Sullivan, D. (2015). Big data in manufacturing: A systematic mapping study. Journal of Big Data, 1, 20.

[8] Susto, G. A., Schirru, A., Pampuri, S., McLoone, S., and Beghi, A. (2015). Machine learning for predictive maintenance: A multiple classifier approach. IEEE Transactions on Industrial Informatics, 11(3):812-820. doi:10.1109/TII.2014.2349359.

[9] https://www.bls.gov/news.release/prod5.nr0.html

[10] Gutenberg, E. (1951):Basics of Business Administration .Volume 1, Berlin u.a.: SpringerVerlag.

[11] Chiang, S. , Kuo, C. , and Meerkov, S. (1998). Bottlenecks in Markovian production lines: A systems approach. IEEE transactions on robotics and automation, 14(2), 352-359.

[12] Scholz-Reiter, B., Katja W. and Liu, H (2011). Modeling dynamic Bottlenecks in Production Networks. International Journal of Computer Integrated Manufacturing. 24(5), 391-404.

[13] Lawrence, S. and Buss, A. (1994). Economic Analysis of Production Bottlenecks. Mathematical Problems in Engineering 1(4). 
[14] Kuo, C., Lim, J. and Meerkov, S. (1996). Bottlenecks in serial production lines: A system theoretic approach. Mathematical problems in engineering, 2.

[15] Li, L., and Ni, J. (2008). Short-term decision support system for maintenance task prioritization. International Journal of Production Economics, 121, 195-202.

[16] Li, L., Chang, Q., and Ni, J. (2009). Data-driven bottleneck detection of manufacturing systems. International Journal of Production Research, 47(18), 5019-5036.

[17] Umble, M. and Srikanth, M. (1990). Synchronous Manufacturing: Principles for World- Class Excellence Cincinnati, OH: South.

[18] Roser, C., Lorentzen, K. and Deuse, J. (2015). Reliable shop floor bottleneck detection for flow lines through process and inventory observations: the bottleneck walk. Logistics Research, 8(1),1-9.

[19] Chang, Q., Ni, J., Bandyopadhyay, P., Biller, S., and Xiao, G. (2007). Supervisory factory control based on real-time production feedback. Journal of Manufacturing Science Engineering, 129(3), 653.

[20] Kolińska, J., and Domański, R. (2017). The Analysis Of Production Lines BottlenecksIdentification And Ways Of Management. Business Logistics in Modern Management.

[21] Konopka, J.(1995). Capacity utilization bottleneck efficiency system-CUBES. IEEE Transactions on Components, Packaging, and Manufacturing Technology: Part A, 18(3), 484-491.

[22] Pegels, C. and Watrous, C. ( 2005), Application of the theory of constraints to a bottleneck operation in a manufacturing plant. Journal of Manufacturing Technology Management. 16(3), 302.

[23] Alden, J., Burns, L., Costy, T., Hutton, R., Jackson, C., Kim, D., and Veen, D. (2006). General Motors increases its production throughput. Interfaces, 36(1), 6-254

[24] Nakata, T., Matsui, K., Miyake, Y. and Nishioka, K. (1999). Dynamic bottleneck control in a wide variety production factory. IEEE Transactions on Semiconductor Manufacturing, 12(3), 273-280. 
[25] Mabin, V. and Balderstone, S. (2003). The performance of the theory of constraints methodology. International Journal of Operations \& Production Management. 23(6), 568595.

[26] MacBryde, J., Radnor, Z., Taj, S. and Berro, L. (2006). Application of constrained management and lean manufacturing in developing best practices for productivity improvement in an auto-assembly plant. International Journal of Productivity and Performance Management. 55(3).

[27] Krishnan, S., Dev, A. S., Suresh, R., Sumesh, A., and Rameshkumar, K. (2018). Bottleneck identification in a tyre manufacturing plant using simulation analysis and productivity improvement. Materials Today: Proceedings, 5(11), 24720-24730.

[28] Baesler, F., Araya, E., Ramis, F. , and Sepúlveda, J. (2004). The use of simulation and design of experiments for productivity improvement in the sawmill industry. In Proceedings of the 2004 Winter Simulation Conference, 2004. (Vol. 2, pp. 1218-1221). IEEE.

[29] Mouzon, G., Yildirim, M. and Twomey, J. (2007). Operational methods for minimization of energy consumption of manufacturing equipment. International Journal of production research, 45(18-19), 4247-4271.

[30] Drake, R., Yildirim, M., Twomey, J., Whitman, L., Ahmad, J. and Lodhia, P. (2006). Data collection framework on energy consumption in manufacturing.

[31] Zhengcai, C., Jijie, D., Min, L., and Yongji, W. (2012). Bottleneck prediction method based on improved adaptive network-based fuzzy inference system (ANFIS) in semiconductor manufacturing system. Chinese Journal of Chemical Engineering, 20(6), 1081-1088.

[32] Lawrence, S. and Buss, A. (1994). Shifting production bottlenecks: causes, cures, and conundrums. Prod. Oper. Manag. 3, 21-37.

[33] Roser, C., Nakano, M., and Tanaka, M. (2002). Detecting shifting bottlenecks.In International Symposium on Scheduling (pp. 59-62).

[34] Wang, J., Chen, J., Wang, S., Zhang, Y. and Sun, S. (2011), December. Shifting economic bottleneck identification. In 2011 IEEE International Conference on Industrial Engineering and Engineering Management (pp. 1760-1764). IEEE 
[35] Tamilselvan, P., Krishnan, K. and Cheraghi, H. (2010). Measurement of shifting bottlenecks in a non-buffer production system. In IIE Annual Conference. Proceedings (p. 1). Institute of Industrial and Systems Engineers (IISE).

[36] Subramaniyan, M., Skoogh, A., Salomonsson, H., Bangalore, P., Gopalakrishnan, M., and Sheikh Muhammad, A. (2018). Data-driven algorithm for throughput bottleneck analysis of production systems. Production \& Manufacturing Research, 6(1), 225-246.

[37] Li, L., Chang, Q., Xiao, G. and Ambani, S. (2011). Throughput bottleneck prediction of manufacturing systems using time series analysis. Journal of Manufacturing Science and Engineering, 133(2).

[38] Moss, H. and Yu, W. (1999). Toward the estimation of bottleneck shiftiness in a manufacturing operation. Production and Inventory Management Journal, 40(2), 53.

[39] Li, R. K., \& Wu, H. H. (1995). Capacity constraint resource wandering and its wandering behaviors in a production plant. Journal of the Chinese Institute of IndustrialEngineers, 12(1), 63-69.

[40] Lu, J., Shen, M., and Lan, X. (2006). Study of the shifting production bottleneck: possible causes and solutions. In 2006 IEEE International Conference on Service Operations and Logistics, and Informatics (pp. 684-688). IEEE.

[41] Chen, L. and Shen, M., (2010). Production bottleneck shiftiness study. In 2010 International Conference on System Science, Engineering Design and Manufacturing Informatization (Vol. 2, pp. 213-216). IEEE.

[42] Glock, C. and Jaber, M. (2013). Learning effects and the phenomenon of moving bottlenecks in a two-stage production system. Applied Mathematical Modelling, 37(18-19), 86178628.

[43] Mouzon, G., and Yildirim, M. (2008). A framework to minimize total energy consumption and total tardiness on a single machine. International Journal of Sustainable Engineering, $1(2), 105-116$.

[44] Chang, Q., Xiao, G., Biller, S. and Li, L. (2012). Energy-saving opportunity analysis of automotive serial production systems (March 2012). IEEE Transactions on Automation Science and Engineering, 10(2), 334-342. 
[45] Thorsten W., Daniel W., Christopher I., and Klaus-Dieter T. (2016) Machine learning in manufacturing: advantages, challenges, and applications, Production \&Manufacturing Research, 4:1, 23-45, DOI: 10.1080/21693277.2016.1192517

[46] Gouarir A., Martínez-Arellano G., Terrazas G., Benardos P. and Ratchev S. (2018). Inprocess Tool Wear Prediction System Based on Machine Learning Techniques and Force Analysis. Procedia CIRP. 77. 501-504.

[47] Chao S., Fan Y., Dexian H., and Wenxiang L. (2014), Data-driven soft sensor development based on deep learning technique, Journal of Process Control, 24(3)., 223-233.

[48] Hsueh, Y. and Yang, C. (2009). Tool breakage diagnosis in face milling by support vector machine. J. Mater. Process. Technol. 209(1), 145-152.

[49] Mukund S, Anders S, Azam S., Bokrantz J., Björn J and Roser C.(2020), A generic hierarchical clustering approach for detecting bottlenecks in manufacturing, Journal of Manufacturing Systems, (55),143-158.

[50] https://llis.nasa.gov/lesson/840

[51] Lei Q and Li T (2017). Identification approach for bottleneck clusters in a job shop based on theory of constraints and sensitivity analysis. Proc Inst Mech Eng Part B J Eng Manuf 2017;231:1091 101.https://doi.org/10.1177/0954405415583884.

[52] Wierzchoń, S., and Mieczysław A (2018). Modern algorithms of cluster analysis. Springer.

[53] Hirano S and Tsumoto S.(2005). Empirical comparison of clustering methods for Long timeseries databases. In: Tsumoto S, Yamaguchi T, Numao M, Motoda H, editors. Act.Min. Berlin Heidelberg: Springer; 2005. p. 275-94. 


\section{Appendix I}

\begin{tabular}{|c|c|c|c|c|c|}
\hline Time & Machine 1 & $\begin{array}{l}\text { Machin } \\
\text { e } 2\end{array}$ & $\begin{array}{l}\text { Machine } \\
3\end{array}$ & $\begin{array}{l}\text { Machine } \\
4\end{array}$ & $\begin{array}{l}\text { Machin } \\
\text { e } 5\end{array}$ \\
\hline 270 & Active & Active & Active & Active & Active \\
\hline 272.5 & Active & Active & Active & Active & Active \\
\hline 275 & Active & Active & Inactive & Active & Active \\
\hline 277.5 & Active & Active & Inactive & Active & Active \\
\hline 280 & Active & Active & Inactive & Active & Active \\
\hline 282.5 & Active & Active & Inactive & Active & Active \\
\hline 285 & Active & Inactive & Active & Active & Active \\
\hline 287.5 & Active & Inactive & Active & Active & Active \\
\hline 290 & Active & Inactive & Active & Active & Active \\
\hline 292.5 & Active & Inactive & Active & Active & Active \\
\hline 295 & Active & Inactive & Active & Active & Active \\
\hline 297.5 & Active & Inactive & Active & Active & Active \\
\hline 300 & Active & Active & Active & Active & Active \\
\hline 302.5 & Active & Active & Active & Active & Active \\
\hline 305 & Active & Active & Inactive & Active & Active \\
\hline 307.5 & Active & Active & Inactive & Active & Active \\
\hline 310 & Active & Active & Inactive & Active & Active \\
\hline 312.5 & Active & Active & Inactive & Active & Active \\
\hline 315 & Active & Inactive & Active & Active & Active \\
\hline 317.5 & Active & Inactive & Active & Active & Active \\
\hline 320 & Active & Inactive & Active & Active & Active \\
\hline 322.5 & Active & Inactive & Active & Active & Active \\
\hline 325 & Active & Inactive & Active & Active & Active \\
\hline 327.5 & Active & Inactive & Active & Active & Active \\
\hline 330 & Active & Active & Active & Active & Active \\
\hline 332.5 & Active & Active & Active & Active & Active \\
\hline 335 & Active & Active & Inactive & Active & Active \\
\hline 337.5 & Active & Active & Inactive & Active & Active \\
\hline 340 & Active & Active & Inactive & Active & Active \\
\hline 342.5 & Active & Active & Inactive & Active & Active \\
\hline 345 & Active & Inactive & Active & Active & Active \\
\hline 347.5 & Active & Inactive & Active & Active & Active \\
\hline 350 & Active & Inactive & Active & Active & Active \\
\hline 352.5 & Active & Inactive & Active & Active & Active \\
\hline 355 & Active & Inactive & Active & Active & Active \\
\hline 357.5 & Active & Inactive & Active & Active & Active \\
\hline 360 & Active & Active & Active & Active & Active \\
\hline 362.5 & Active & Active & Active & Active & Active \\
\hline
\end{tabular}

